\title{
A PRELIMINARY COLOR-MAGNITUDE DIAGRAM FOR THE URSA MINOR DWARF GALAXY
}

\author{
Robert A. Schommer and Edward W. Olszewski \\ University of Washington \\ William E. Kunkel \\ Observatorio Nacional de Brasil
}

The Ursa Minor dwarf galaxy ( $\mathrm{RA}=15^{\mathrm{h}} 08^{\mathrm{m}} \cdot 2$, Dec $=+67^{\circ} 18^{\prime}(1950)$ ) was one of four dwarf ellipticals discovered by Wilson (1955) in a search of the Palomar Sky survey plates. The general properties of these galaxies resemble those of galactic globular clusters, linear size and density excepted (see Hodge, 1971, for a review). The characteristics of Ursa Minor are very similar to those of the Draco dwarf, for which a color magnitude diagram was published by Baade and Swope (1961). Van Agt (1967) published a study of the variable stars in Ursa Minor based on Baade's 200-inch plates.

Recent studies of distant globular clusters and dwarf galaxies have used these systems as probes of the galactic halo. Studies of the metallicity of individual stars in these systems based on spectra (Cowley, Hartwick and Sargent, 1977) and on photometry (Canterna and Schommer, 1977) have shown that they are extremely metal poor (similar to M92) with some evidence for [CNO] enrichment. The low stellar densities and lack of continual star formation imply an absence of extensive chemical processing in these systems, and thus their current conditions and abundances may reflect those of the proto-Galactic cloud (Eggen, Lynden-Be11, and Sandage, 1962). Most of these systems, including Draco, show evidence for anomalous red horizontal branches (Sandage and Wildey, 1967) and this has led to investigations of possible [He] or age differences (Hartwick, 1968; Rood, 1973). The unique spatial distribution of these systems has inspired Magellanic Plane models (Kunkel and Demers, 1977; Lynden-Bell, 1976). 


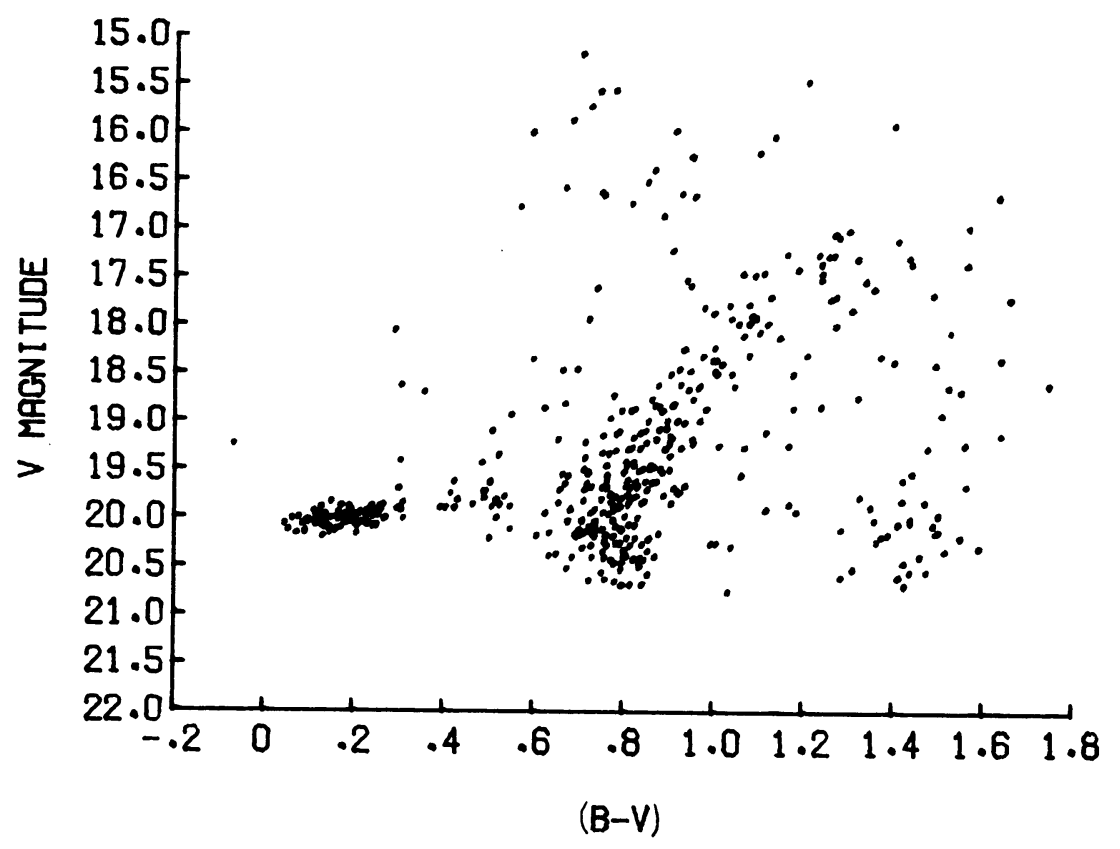

Fig. 1. CM diagram for Ursa Minor, 457 stars measured, excluding variables identified by Van Agt (1967)

Data for the present investigation consist of two prime focus plate pairs taken on the 4-meter Mayall telescope at KPNO, and photometry of standard stars done on the 2.1 meter telescope at KPNO and on the University of Washington $76 \mathrm{~cm}$ telescope. The standard sequence (down to $V=18^{\mathrm{m}} \cdot 7$ ) was extended to $\mathrm{V}=22^{\mathrm{m}} \cdot 0$ on the plates through the use of a Racine wedge (Racine, 1969) which forms secondary images $6.91( \pm .05)$ magnitudes fainter than the primary. This calibration was checked on the standard sequence of $M 13$.

Parameters derived from this color magnitude diagram (Fig. 1) are $\Delta V=2^{m} .9 \pm 0.2, S=5.7 \pm 0.4$, and $(B-V)_{0, g}=0.96 \pm 0 m 04$. The se results and the blue horizontal-branch morphology indicate a metal poor system, and agree well with the value of $[\mathrm{Fe} / \mathrm{H}]=-2.1 \mathrm{re}-$ cently determined by Canterna and Schommer (1977). The reddening, determined by UBVr field star photometry and the blue edge of the $R R$ Lyrae gap, is found to be $E(B-V)=0.06 \pm .03$. By assuming $M_{\mathrm{v}}=0.6$ for the RR Lyrae stars, we derive $\bar{a}$ true distance modulus of $19.2(69 \mathrm{kpc})$. The color magnitude diagram looks remarkably like that of M92 and the luminosity function resembles that of M3 (Sandage 1954). 
Ursa Minor exhibits a horizontal-branch morphology different than the other halo objects and dwarf galaxies. Essentially all the systems with $\mathrm{R}_{\mathrm{gc}}>20 \mathrm{kpc}$ show the red horizontal-branch anomaly and several of the galaxies have unusual variable stars (Swope 1968). The postulated Magellanic Plane tied these systems together and possibly pointed to a different epoch of formation. The seemingly "normal" properties of the Ursa Minor system, however, complicate this interesting explanation and the recent radial velocity determinations (Hartwick and Sargent 1977) seriously damage the dynamical model of Lynden-Bell (1976).

The similarity between the extreme Population II globular clusters and Ursa Minor has implications for pre-Galactic element enrichment. Hodge and Mitchie (1969) have argued that Ursa Minor formed much farther from the Galaxy than its present position, so the apparent uniformity of chemical abundances implies the same or at least very similar initial enrichments over a spatial scale of several hundred kpcs.

\section{REFERENCES}

Baade, W. and Swope, H. (1961). Astron. J. 66, 300. Cowley, A., Hartwick, F.D.A. and Sargent, W.L.W. (1977). preprint. Canterna, R. and Schommer, R. (1977). in press.

Eggen, O., Lynden-Bell, D. and Sandage, A. (1962). Astrophys. J. 136,748 .

Hartwick, F.D.A. (1968). Astrophys. J. 154, 475.

Hartwick, F.D.A. and Sargent, W.L.W. (1977). preprint.

Hodge, P. (1971). Ann. Rev. of Astron. and Astrophys. 9, 35.

Hodge, P. and Mitchie, R. (1969). Astron. J. 74, 587.

Kunkel, W. and Demers, S. (1976). Greenwich Jbs. Bulletin \#182.

Lynden-Bell, D. (1976). Monthly Notices of the R.A.S. 174, 695.

Racine, R. (1969). Astron. J. 74, 1073.

Rood, R. (1973). Astrophys. J. 184, 815.

Sandage, A. $(1 9 5 4 \longdiv { \text { . Astron.J. } } . 5 9,192$.

Sandage, A. and Wildey, R. (1967). Astrophys. J. 150, 469.

Swope, H. (1968). Astron. J. 73, 2504.

Van Agt, S.L. Th. (1967). Bull. Astron. Neth. 19, 275.

Wilson, A. (1955). Publ. Astron. Soc. Pacific 67, 27. 


\section{DISCUSSION}

TINSLEY: You remarked at the end that this system gives information on the scale of chemical enrichment. Can you say more about the homogeneity of abundances in the UMi system?

SCHOMMER: Not really at this preliminary stage. The diagram here samples 2500 stars in the central field surveyed for variables by van Agt. We are in the process of reducing a separate field in the outer regions of this galaxy. I think the width of the giant branch in our diagram here can be attributed to observational error and field star contamination. 